\title{
Energy Transfer into Molecular Vibrations and Rotations by Recoil in Inner-Shell Photoemission
}

\author{
E. Kukk, ${ }^{1}$ T. D. Thomas, ${ }^{2}$ D. Céolin, ${ }^{3}$ S. Granroth, ${ }^{1}$ O. Travnikova, ${ }^{4}$ M. Berholts, ${ }^{1,5}$ T. Marchenko, ${ }^{4}$ R. Guillemin,${ }^{4}$ \\ L. Journel, ${ }^{4}$ I. Ismail,${ }^{4}$ R. Püttner, ${ }^{6}$ M. N. Piancastelli, ${ }^{4,7}$ K. Ueda, ${ }^{8}$ and M. Simon ${ }^{4,3}$ \\ ${ }^{1}$ Department of Physics and Astronomy, University of Turku, FI-20014 Turku, Finland \\ ${ }^{2}$ Department of Chemistry, Oregon State University, Corvallis, Oregon 97331, USA \\ ${ }^{3}$ Synchrotron SOLEIL, L'Orme des Merisiers, Saint-Aubin, B.P. 48, FR-91192 Gif-sur-Yvette Cedex, France \\ ${ }^{4}$ Sorbonne Université, CNRS, Laboratoire de Chimie Physique-Matière et Rayonnement, LCPMR, F-75005 Paris, France \\ ${ }^{5}$ Department of Physics, University of Tartu, EST-50411 Tartu, Estonia \\ ${ }^{6}$ Fachbereich Physik, Freie Universität Berlin, Arnimallee 14, D-14195 Berlin-Dahlem, Germany \\ ${ }^{7}$ Department of Physics and Astronomy, Uppsala University, SE-75120 Uppsala, Sweden \\ ${ }^{8}$ Institute of Multidisciplinary Research for Advanced Materials, Tohoku University, Sendai 980-8577, Japan
}

(Received 18 May 2018; published 17 August 2018)

\begin{abstract}
A mixture of $\mathrm{CF}_{4}$ and $\mathrm{CO}$ gases is used to study photoelectron recoil effects extending into the tender $\mathrm{x}$ ray region. In $\mathrm{CF}_{4}$, the vibrational envelope of the $\mathrm{C} 1 s$ photoelectron spectrum becomes fully dominated by the recoil-induced excitations, revealing vibrational modes hidden from Franck-Condon excitations. In $\mathrm{CO}$, using $\mathrm{CF}_{4}$ as an accurate energy calibrant, we determine the partitioning of the recoil-induced internal excitation energy between rotational and vibrational excitation. The observed rotational recoil energy is 2.88(28) times larger than the observed vibrational recoil energy, well in excess of the ratio of 2 predicted by the basic recoil model. The experiment is, however, in good agreement with the value of 2.68 if energy transfer via Coriolis coupling is included.
\end{abstract}

DOI: $10.1103 /$ PhysRevLett.121.073002

Electron spectroscopic studies of photoemission from the atomic inner shells provide valuable information on the electronic states and potential energy surfaces of molecules as well as on the nuclear dynamics accompanying photoionization. In particular, the experimental determination of the peak intensities in the vibrational profile allows one to determine the Franck-Condon factors and assess the shape of the potential energy surfaces. The accurate determination of both the vibrational profiles and the ionization energies is essential for extracting correct chemical information. However, these quantities are also affected by other phenomena such as photoelectron recoil [1-5] and diffraction [6-8], which have often not been properly accounted for. Furthermore, photoelectron diffraction and recoil effects can themselves be a valuable source of information about the molecular environment or be a source of novel features observed in photoelectron and even atomic Auger electron spectra [9].

Photoelectron recoil can lead to vibrational excitation, as has been observed in the carbon $1 s$ ionization of $\mathrm{CH}_{4}$ [1], graphite [10], and $\mathrm{CF}_{4}$ [2], and rotational excitation, as seen in the valence ionization of $\mathrm{N}_{2}[3,4]$. However, no measurements have been reported in which both vibrational and rotational excitation have been observed and quantified simultaneously, and recoil-induced rotational excitation during core ionization has not been observed at all. Although here we present such results for carbon $1 \mathrm{~s}$ ionization of gas-phase $\mathrm{CO}$, it should be noted that recoil-induced excitations are present also in solids $[10,11]$ and are especially prominent in hard $\mathrm{x}$ ray photoelectron spectroscopy experiments.

The division of the internal excitation between vibration and rotation is influenced by the angular distribution of the photoelectrons with respect to the molecular axis. For a linear molecule, the component of momentum along the molecular axis leads to vibrational excitation, and the component perpendicular to the axis leads to rotational excitation. The corresponding recoil-induced vibrational and rotational excitation energies go as $\left\langle\cos ^{2} \theta\right\rangle$ and $\left\langle\sin ^{2} \theta\right\rangle$, respectively, where $\theta$ is the angle of emission of the photoelectron with respect to the molecular axis. For isotropic emission from a diatomic molecule (which we expect for $\mathrm{CO}$ at high photoelectron energies), the division should be $2 / 3$ to rotation and $1 / 3$ to vibration [3]. However, carbon $1 s$ ionization of $\mathrm{CO}$ results in a shrinkage of the $\mathrm{CO}$ bond length between the neutral molecule and the core-ionized molecule [12]. Because of this shrinkage, it is predicted that Coriolis coupling will lead to a transfer of energy from the vibrational mode to the rotational mode [13]. Until now, this predicted effect has not been observed.

By the expression "recoil momentum," we refer to the momentum that remains in the ion after the photoelectron has been ejected $[14,15]$. The photoelectron (energy equal to $\epsilon_{e}$ ) has a momentum $p_{e}=\sqrt{2 \epsilon_{e} m_{e}}\left(m_{e}\right.$ is the electron 
mass). Since the photon carries insignificant momentum, the electron ejected must be one that had the appropriate momentum within the molecule. Correspondingly, the remaining ion must have had equal and opposite momentum.

Photoelectrons with a momentum corresponding to a kinetic energy of $\gtrsim 10 \mathrm{eV}$ must originate close to the nucleus of one of the atoms - the emitter atom $A$-and the expectation is that their recoil momentum will be associated with the atom $A$. This is the main assumption of the recoil model applied here-that the recoil momentum can initially be associated with a single atom. This is equivalent to the assumption that the characteristic timescale of molecular vibrations is much longer than that of the electron motion. The distribution of this single-atom recoil momentum among the normal modes of the entire molecule can be obtained by projecting it onto all of the normal mode momentum vectors of the molecule-translational, rotational, and vibrational. The translational recoil is the most trivial form, where the translational recoil energy is determined by the momentum conservation as $p_{e}^{2} /(2 M)$ (with $M$ as the molecular mass), but is less than the total recoil energy $p_{e}^{2} /\left(2 M_{A}\right)$, where $M_{A}$ is the mass of the emitter atom. The energy difference excites the internal (rotational and vibrational) degrees of freedom (d.o.f.).

Increasingly more accurate experimental data on the photoelectron recoil effect have become available in the past decade, and, concurrently, theoretical models of the photoelectron recoil have also evolved to quite elaborate calculations of the intensity ratios of the vibrational peaks. A boost to these studies has come from moving from the soft $\mathrm{x}$ ray regime to the tender $\mathrm{x}$ rays, since the strength of the photoelectron recoil effects increases with the kinetic energy of the emitted electron.

In earlier investigations, an important goal was to develop more accurate quantum mechanical models to describe the recoil excitation probabilities of individual vibrational energy levels [2,16-18]; here we will focus on the general description in terms of energy deposition into various d.o.f. The prediction of the total amount of recoil energy that goes into the internal d.o.f. is quite robust, requiring no other assumptions than the one mentioned above. The prediction of the division of the internal energy into the rotational and vibrational d.o.f. is, however, model dependent, and accurate model predictions are still being developed. For example, the anisotropy of the photoemission in the molecular frame can alter this separation, as can the changes in the equilibrium bond length upon ionization.

Here, we present direct experimental data on the partitioning of the recoil energy into rotational and vibrational excitations in the $\mathrm{CO}$ molecule and on the role of Coriolis coupling on this partition. We report measurements on the photoelectron recoil in $\mathrm{C} 1 s$ photoemission from carbon monoxide over an extended energy range up to $\approx 7 \mathrm{keV}$; these are complete in the sense that all the components of the recoil excitations have been determined. This requires accurate electron kinetic energy calibration, which was achieved using a gas mixture of $\mathrm{CO}$ and $\mathrm{CF}_{4}$. Carbon tetrafluoride is in several ways a good calibrant in photoelectron recoil studies, as will emerge in the discussion below. $\mathrm{CF}_{4}$ is also an excellent target for studying vibrational recoil excitations. First, we will present a detailed study of photoelectron recoil vibrational excitations in $\mathrm{CF}_{4}$ extending into the tender $\mathrm{x}$ ray regions. We then proceed to the complete recoil study of $\mathrm{CO}$ with $\mathrm{CF}_{4}$ as the calibrant.

Carbon tetrafluoride $\left(\mathrm{CF}_{4}\right)$ has tetrahedral symmetry with the carbon atom at its center of mass. Therefore, the departure of the $\mathrm{C} 1 \mathrm{~s}$ photoelectron does not create recoil angular momentum in the residual molecular ion and therefore does not excite rotational d.o.f. All internal recoil excitation energy goes into molecular vibrations, as discussed by Thomas et al. [2]. In that soft x ray study, the amount of vibrational recoil energy was still quite small, less than $40 \mathrm{meV}$.

The results presented here were obtained at the SOLEIL Synchrotron, France, on the GALAXIES beam line equipped with an end station dedicated to hard and tender $\mathrm{x}$ ray photoelectron spectroscopy $[19,20]$. Linearly polarized light is provided by a $\mathrm{U} 20$ undulator and monochromatized by a $\mathrm{Si}(111)$ double crystal monochromator. The photoelectron spectra of the sample gases and their mixture were recorded by a EW4000 Scienta analyzer, mounted with the lens axis collinear with the polarization vector of the $\mathrm{x}$ rays. The spectrometer was operated at $100 \mathrm{eV}$ pass energy and with the entrance slit of $0.3 \mathrm{~mm}$, except for the photon energies of 6900 and $8500 \mathrm{eV}$, at which the larger slit of $0.5 \mathrm{~mm}$ was used. At 6900 and $8500 \mathrm{eV}$, also the third diffraction order was used for enhanced resolution. The $\mathrm{CO}$ and $\mathrm{CF}_{4}$ gases were mixed externally in the gas inlet manifold.

Figure 1 displays a series of $\mathrm{C} 1 s$ photoelectron spectra from the $\mathrm{CF}_{4}$ molecule, recorded between $330 \mathrm{eV}$ and $8 \mathrm{keV}$ photon energy. The first in the series, a nearthreshold spectrum, is reproduced from Thomas et al. [2]. Because of the low photoelectron kinetic energy, the recoil vibrational excitations are negligible in spectrum (a), and also no Franck-Condon excitations are present, since the $\mathrm{C} 1 s$ core ionization is not accompanied by a noticeable geometry change in this molecule [12]. The spectrum therefore consists of a single Voigt profile, but this is strongly distorted by the postcollision interaction (PCI) between the outgoing photo- and Auger electrons. PCI is manifested as peak asymmetry with the tail on the highionization-energy side.

The rest of the spectra in Fig. 1 exhibit pronounced vibrational excitations due to photoelectron recoil. The dominant normal mode excited by the recoil is the asymmetric stretching mode, acquiring over $90 \%$ of the vibrational recoil energy. The rest of the excitation energy goes into the asymmetric bending mode [2]. The instrumental 


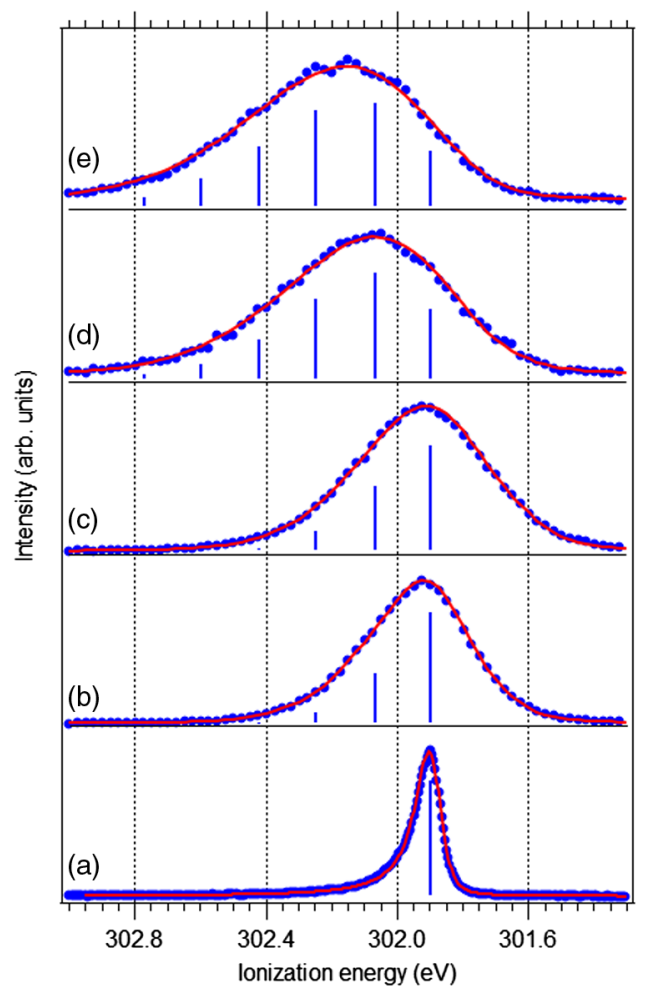

FIG. 1. Carbon $1 s$ photoelectron spectra of $\mathrm{CF}_{4}$, measured at different photon energies: (a) $330 \mathrm{eV}$, (b) $2.3 \mathrm{keV}$, (c) $3.0 \mathrm{keV}$, (d) $6.9 \mathrm{keV}$, and (e) $8.5 \mathrm{keV}$. Dots, experimental data points; continuous red line, least-squares curve-fitting result; vertical sticks, positions and relative intensities of the vibrational peaks.

resolution combined with Doppler broadening in the highenergy spectra [Figs. 1(b)-1(e)] is larger than the vibrational frequency of the asymmetric stretching mode (about $170 \mathrm{meV}$ ) [2], and thus the individual vibrational peaks cannot be directly resolved. Therefore, a least-squares curvefitting analysis of the vibrational envelope was carried out, as described in detail in Supplemental Material [21].

The analysis shows that a satisfactory representation of vibrational recoil excitations in $\mathrm{CF}_{4}$ can been made using a simple-to-apply Poisson distribution approach. Figure 1 shows the fitted envelopes obtained from a single Poisson progression model. The figure also shows the peak positions and intensities for the single normal mode - the asymmetric stretch-used for fitting. The vibrational frequency obtained from the fit was 167(4) $\mathrm{meV}$, in good agreement with the previously reported experimental value of 169(1) eV [2].

The spectra in Fig. 1 represent the first observed case where the entire vibrational profile of a free molecule is due to strong photoelectron recoil effects, causing major changes in the spectral shape and a large shift in its centerof-mass position, rather than relatively subtle modifications observed in earlier studies at lower energies [1,2]. Also, the Poisson fitting procedure allows one to accurately determine the energy of the $\nu=0$ vibrational peak and use $\mathrm{CF}_{4}$ as a calibrant for other samples.
Recoil-induced vibrational excitations in the $\mathrm{CO}$ molecule have been recently analyzed in the energy range up to $7 \mathrm{keV}$ [18]. In CO, the vibrational excitations account only for about one-third of the internal recoil energy, the rest inducing rotational excitations. Although of the same origin, manifestations of the vibrational and rotational recoil in the core-level molecular photoemission spectra are substantially different. An increase of the vibrational energy can be observed as excitations of discrete vibrational levels, causing changes in the peak intensity ratios ( $v$ ratios) within the vibrational envelope. The separation of rotational levels, on the other hand, is so small that rotational excitations can be observed only as a shift of the centroids of each individual peak towards a lower kinetic energy, equal to the average amount of recoil energy deposited into rotations. These recoil shifts have hitherto not been determined in core-level photoemission.

In order to accurately measure the rotational recoil energy shifts in $\mathrm{CO}$, the $\nu=0$ peak position of the $\mathrm{CF}_{4}$ $\mathrm{C} 1 s$ electrons is used as an energy reference, since the $\mathrm{CF}_{4}$ spectrum is unaffected by the rotational recoil. Figure 2 shows the $\mathrm{C} 1 s$ photoelectron spectra measured from a gas mixture of the $\mathrm{CF}_{4}$ calibrant and $\mathrm{CO}$. The energy separation of the $\nu=0$ peaks from both species is the value to be determined for obtaining the rotational recoil energy shift.

The vibrational progressions of both species are represented by appropriate curve-fitting models-see Supplemental Material [21] for details. The energy separations of the two $\nu=0$ peaks were obtained from the fits of the experimental spectra. These separations are indicated by the double-ended red arrows in Fig. 2 for the photon
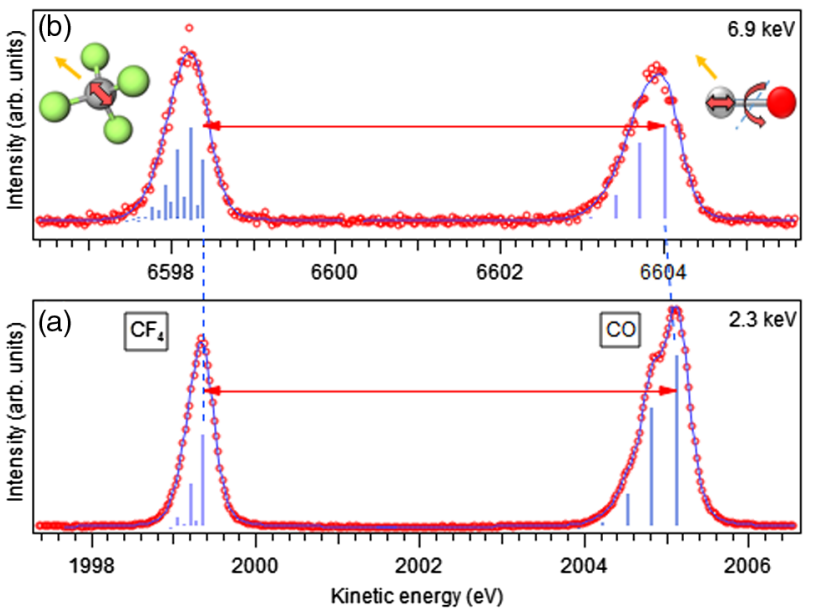

FIG. 2. Carbon $1 s$ photoelectron spectra from a mixture of $\mathrm{CO}$ and $\mathrm{CF}_{4}$ gases, measured at (a) 2.3 and (b) $6.9 \mathrm{keV}$ photon energy. The fitted positions and relative intensities of the vibrational peaks are marked by vertical lines, and the fitted total curves are shown by solid blue lines. Arrows indicate the distance between the $\nu=0$ peaks of $\mathrm{CF}_{4}$ and $\mathrm{CO}$. Dashed lines connect the positions of the $\nu=0$ peaks in both spectra. The $x$-axis energy ranges are chosen to align the $\nu=0$ peaks of $\mathrm{CF}_{4}$. 
energies of 2.3 and $6.9 \mathrm{keV}$. In addition, a similar spectrum at $3.0 \mathrm{keV}$ photon energy was measured and analyzed. The separation includes the difference in the $\mathrm{C} 1 s$ adiabatic ionization energies of the two molecules. A linear regression through the data points (peak separation versus electron kinetic energy) yielded the $y$-axis intercept at $5.828(4) \mathrm{eV}$ for the vertical ionization energy difference, a value in excellent agreement with the literature value of $5.829 \mathrm{eV}$ [22]. The constant offset of $5.828 \mathrm{eV}$ was then subtracted to obtain the part of this shift that depends on the photoelectron kinetic energy.

The separation of the $\nu=0$ peaks for the two molecules is $152(5) \mathrm{meV}$ less at $6.9 \mathrm{keV}$ than it is at $2.3 \mathrm{keV}$. This decrease is readily visible in Fig. 2. Part of this decrease is accounted for by translational recoil. The $\nu=0$ peaks of both molecules are shifted towards a lower kinetic energy due to the translational recoil by the amount of $p_{e l}^{2} /(2 M)$. $\mathrm{CO}$, as the lighter molecule, has a larger translational recoil shift, and the net effect is to lower the separation of the two peaks by $61.5 \mathrm{meV}$ over this energy range. The remaining 90(5) $\mathrm{meV}$ change is assigned to rotational recoil excitation, which is present in $\mathrm{CO}$ but not in $\mathrm{CF}_{4}$.

A similar analysis was done for the $h \nu=3.0 \mathrm{keV}$ spectrum. The three data points for the rotational recoil shifts, representing the average recoil energy going into the rotational motion, are shown in Fig. 3 by the red markers. Their error bars represent both the statistical uncertainly of the analysis and the variations when different curve-fitting scenarios were used to obtain the $\nu=0$ peak energies, e.g., single or double Poisson series and fixed or free Lorentzian width. A linear regression through these data points yields the slope of $1.96(11) \times 10^{-5}$.

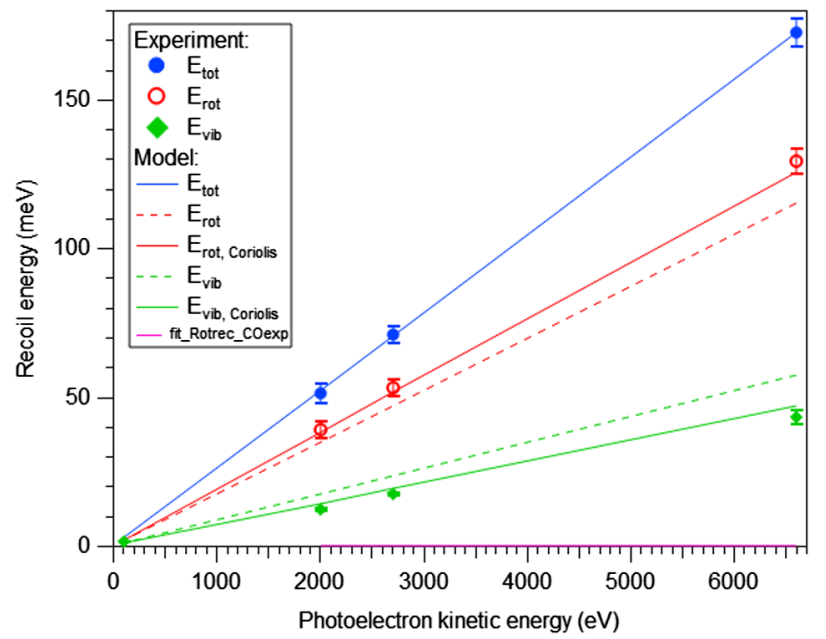

FIG. 3. Recoil energy partitioning into the total internal, rotational, and vibrational recoil excitations. Experiment: solid blue circles, total recoil energy; open red circles, rotational recoil; green diamonds, vibrational recoil. Recoil-model prediction including Coriolis coupling: solid lines; from highest to lowest, total, rotational, and vibrational recoil. Model prediction without Coriolis coupling: dashed lines.
Figure 3 also shows as the green points the average recoil energy going into the vibrational d.o.f. of $\mathrm{CO}$. The average vibrational energy in each $\mathrm{C} 1 s$ photoelectron spectrum arises from both the Franck-Condon and recoil excitations, the former being independent of photon and electron kinetic energy. Changes in the vibrational energy are due to the energy-dependent photoelectron recoil and can be measured directly as changes in the centroid position of the vibrational envelope. In the analysis, the centroid positions were obtained as intensity-weighted averages of the positions of the fitted vibrational peaks. In order to obtain the absolute values of the vibrational recoil energies, rather than the energy changes, a spectrum measured at $h \nu=$ $400 \mathrm{eV}$ from Ref. [8] was reanalyzed to obtain the centroid energy under the conditions of very weak recoil. Linear regression through all four data points $(0.4,2.3,3.0$, and $6.9 \mathrm{keV}$ ) then gave the zero-kinetic-energy intercept at $168 \mathrm{meV}$ centroid energy, corresponding to the "pure" Franck-Condon excitations. This value is in agreement with the value of $160 \mathrm{meV}$ reported by Myrseth et al. [22]. The value of $168 \mathrm{meV}$ was subtracted from the vibrational centroid energies of $\mathrm{CO}$ before plotting them in Fig. 3. The shift of the centroid energy, with the slope of $6.8(5) \times 10^{-6}$, is due to the vibrational recoil.

The total recoil energy (blue markers in Fig. 3) is given as the sum of the vibrational and rotational recoil energies. A straight line fit to these points has a slope of $2.63(12) \times 10^{-5}$, which is in good agreement with the predicted slope of $2.612 \times 10^{-5}$ (solid blue line in Fig. 3). The various recoil energies extracted from the spectra at the three photon energies are collected in Table I.

The ratio of the two slopes measured for the rotational and vibrational excitation is 2.88(28), and this gives the partitioning of the recoil-induced internal energy between the rotational and vibrational modes. It disagrees with the ratio of 2 (exact) predicted using the basic recoil model and assuming isotropic molecular-frame angular distribution of the photoelectron. The basic model predictions are shown in Fig. 3 by the red (rotational recoil energy) and green (vibrational recoil energy) dashed lines. However, this prediction does not take into account the transfer of energy from vibrational to rotational excitation through Coriolis interaction. The equilibrium bond length of $\mathrm{CO}$ shortens upon core ionization, which leads to a lower moment of inertia and, hence, to a higher rotational energy for a given angular momentum. This increase in rotational energy is

TABLE I. Energy separations of the $\nu=0$ vibrational levels of $\mathrm{CO}$ and $\mathrm{CF}_{4}$, the rotational, vibrational, and total internal recoil experimentally determined excitation energies of $\mathrm{CO}$.

\begin{tabular}{lcccr}
\hline \hline$h \nu(\mathrm{eV})$ & $\Delta E(\mathrm{eV})$ & $E_{\text {rot }}(\mathrm{meV})$ & $E_{\text {vib }}(\mathrm{meV})$ & $E_{\text {tot }}(\mathrm{meV})$ \\
\hline 2300 & $5.762(3)$ & $39(3)$ & $12.3(0.7)$ & $51(3)$ \\
3000 & $5.738(3)$ & $53(3)$ & $17.6(0.5)$ & $71(3)$ \\
6900 & $5.610(4)$ & $129(4)$ & $43.3(2.4)$ & $172(5)$ \\
\hline \hline
\end{tabular}


taken from the vibrational excitation through Coriolis coupling [13]. In $\mathrm{CO}$, the bond length shortens from 1.128 to $1.079 \AA$ [8], which results in a Coriolis-induced increase in the rotational recoil energy by $9.3 \%$ and a decrease by the same amount (and percentage of 18.6\%) in the vibrational recoil energy. Including Coriolis coupling in the model increases the predicted ratio of rotational to vibrational energy from 2.00 to 2.68 (shown by the solid red and green lines in Fig. 3) that is in very good agreement with the experiment.

In summary, we have investigated photoelectron-recoilinduced internal excitations in two molecules with a very different response-in $\mathrm{CF}_{4}$, recoil causes major changes in the $\mathrm{C} 1 s$ vibrational profile as the ionizing photon energy is extended into the tender $\mathrm{x}$ ray regime; in $\mathrm{CO}$, most of the recoil energy is delivered to the molecular rotations, causing apparent shifts in the observed kinetic energies of the $\mathrm{C} 1 s$ photoelectron spectrum. A convenient approach, based on a Poisson distribution, has been used in analyzing the vibrational recoil effects in $\mathrm{CF}_{4}$ and is shown to model the observed spectral structures with good accuracy. The $\mathrm{C} 1 s$ photoelectron spectra of $\mathrm{CF}_{4}$ represents the first observation of an entire vibrational envelope with excitations up to at least the $\nu=4$ level that are entirely caused by photoelectron recoil, whereas in the soft $\mathrm{x}$ ray region typically only recoil excitations by a single vibrational quantum can be observed. Such a recoil-excited vibrational envelope reveals vibrational modes that are not populated by normal Franck-Condon excitation, and, by extracting the vibrational frequency of the asymmetric stretch mode, we have demonstrated how recoil excitations can be used to probe otherwise inaccessible regions and properties of the molecular potential energy surfaces.

Based on the above analysis, we have developed a method of using $\mathrm{CF}_{4}$ as a calibrant in the recoil energy analysis of other molecules. Applying this approach to $\mathrm{CO}$, a complete recoil energy analysis has been performed, covering simultaneously both the rotational and vibrational degrees of freedom for the first time. By comparing the experimentally determined energy partitioning ratio with model predictions, we have observed a significantly higher fraction of energy going into the rotational excitations than predicted by the basic recoil model. However, applying a model that includes Coriolis coupling gives an excellent agreement with the experiment, thus providing the first experimental confirmation of the predicted role of the Coriolis coupling in photoelectron recoil.

The authors thank the GALAXIES team for their help with the experiment. E. K. acknowledges funding from the Academy of Finland. K. U. acknowledges support for the XFEL strategy funding by MEXT, the five star alliance, and the TAGEN project. M. B. acknowledges the ERDF project High-technology Materials for Sustainable Development TK117.
[1] E. Kukk, K. Ueda, U. Hergenhahn, X.-J. Liu, G. Prümper, H. Yoshida, Y. Tamenori, C. Makochekanwa, T. Tanaka, M. Kitajima, and H. Tanaka, Phys. Rev. Lett. 95, 133001 (2005).

[2] T. D. Thomas, E. Kukk, R. Sankari, H. Fukuzawa, G. Prümper, K. Ueda, R. Püttner, J. Harries, Y. Tamenori, T. Tanaka, M. Hoshino, and H. Tanaka, J. Chem. Phys. 128, 144311 (2008).

[3] T. D. Thomas, E. Kukk, H. Fukuzawa, K. Ueda, R. Püttner, Y. Tamenori, T. Asahina, N. Kuze, H. Kato, M. Hoshino, H. Tanaka, M. Meyer, J. Plenge, A. Wirsing, E. Serdaroglu, R. Flesch, E. Rühl, S. Gavrilyuk, F. Gel'mukhanov, A. Lindblad et al., Phys. Rev. A 79, 022506 (2009).

[4] T. D. Thomas, E. Kukk, T. Ouchi, A. Yamada, H. Fukuzawa, K. Ueda, R. Püttner, I. Higuchi, Y. Tamenori, T. Asahina, N. Kuze, H. Kato, M. Hoshino, H. Tanaka, A. Lindblad, and L. J. Sæthre, J. Chem. Phys. 133, 174312 (2010).

[5] Y. Krivosenko and A. Pavlychev, Chem. Phys. Lett. 664, 233 (2016).

[6] J. Söderström, N. Mårtensson, O. Travnikova, M. Patanen, C. Miron, L. J. Sæthre, K. J. Børve, J. J. Rehr, J. J. Kas, F. D. Vila, T. D. Thomas, and S. Svensson, Phys. Rev. Lett. 108, 193005 (2012).

[7] K. Ueda, C. Miron, E. Plésiat, L. Argenti, M. Patanen, K. Kooser, D. Ayuso, S. Mondal, M. Kimura, K. Sakai, O. Travnikova, A. Palacios, P. Decleva, E. Kukk, and F. Martín, J. Chem. Phys. 139, 124306 (2013).

[8] E. Kukk, D. Ayuso, T. D. Thomas, P. Decleva, M. Patanen, L. Argenti, E. Plésiat, A. Palacios, K. Kooser, O. Travnikova, S. Mondal, M. Kimura, K. Sakai, C. Miron, F. Martín, and K. Ueda, Phys. Rev. A 88, 033412 (2013).

[9] M. Simon, R. Püttner, T. Marchenko, R. Guillemin, R. K. Kushawaha, L. Journel, G. Goldsztejn, M. N. Piancastelli, J. M. Ablett, J.-P. Rueff, and D. Céolin, Nat. Commun. 5, 4069 (2014).

[10] Y. Takata, Y. Kayanuma, M. Yabashi, K. Tamasaku, Y. Nishino, D. Miwa, Y. Harada, K. Horiba, S. Shin, S. Tanaka, E. Ikenaga, K. Kobayashi, Y. Senba, H. Ohashi, and T. Ishikawa, Phys. Rev. B 75, 233404 (2007).

[11] S. Suga, A. Sekiyama, H. Fujiwara, Y. Nakatsu, T. Miyamachi, S. Imada, P. Baltzer, S. Niitaka, H. Takagi, K. Yoshimura, M. Yabashi, K. Tamasaku, A. Higashiya, and T. Ishikawa, New J. Phys. 11, 073025 (2009).

[12] T. X. Carroll, K. J. Børve, L. J. Sæthre, J. D. Bozek, E. Kukk, J. A. Hahne, and T. D. Thomas, J. Chem. Phys. 116, 10221 (2002).

[13] T. D. Thomas, Phys. Rev. A 90, 052504 (2014).

[14] E. Kukk, T. D. Thomas, and K. Ueda, J. Electron Spectrosc. Relat. Phenom. 183, 53 (2011).

[15] E. Kukk, K. Ueda, and C. Miron, J. Electron Spectrosc. Relat. Phenom. 185, 278 (2012).

[16] W. Domcke and L. Cederbaum, J. Electron Spectrosc. Relat. Phenom. 13, 161 (1978).

[17] F. Gel'mukhanov, P. Salek, and H. Ågren, Phys. Rev. A 64, 012504 (2001).

[18] E. Kukk, T. D. Thomas, K. Ueda, D. Céolin, S. Granroth, K. Kooser, O. Travnikova, D. Iablonsky, P. Decleva, D. Ayuso, R. Püttner, H. Levola, G. Goldsztejn, T. Marchenko, M. N. Piancastelli, and M. Simon, Phys. Rev. A 95, 042509 (2017). 
[19] D. Céolin, J. M. Ablett, D. Prieur, T. Moreno, J.-P. Rueff, T. Marchenko, L. Journel, R. Guillemin, B. Pilette, T. Marin et al., J. Electron Spectrosc. Relat. Phenom. 190, 188 (2013).

[20] J.-P. Rueff, J. M. Ablett, D. Céolin, D. Prieur, T. Moreno, V. Balédent, B. Lassalle-Kaiser, J. Rault, M. Simon, and A. Shukla, J. Synchrotron Radiat. 22, 175 (2015).
[21] See Supplemental Material at http://link.aps.org/ supplemental/10.1103/PhysRevLett.121.073002 for details about the spectral analysis using least-squares curve fitting and the extraction of peak positions and intensities.

[22] V. Myrseth, J. D. Bozek, E. Kukk, L. J. Sæthre, and T. D. Thomas, J. Electron Spectrosc. Relat. Phenom. 122, 57 (2002). 